\title{
ON A 4-MANIFOLD HOMOLOGY EQUIVALENT TO A BOUQUET OF SURFACES ${ }^{1}$

\author{
BY
}

\author{
AKIO KAWAUCHI
}

\begin{abstract}
This paper gives some algebraic invariants for a piecewise linear imbedding of a surface into some 4-manifold inducing a $Z$ or $Q$-homology isomorphism. Several examples are obtained by using these invariants.
\end{abstract}

Let $F$ be a closed (possibly disconnected) oriented surface and let $W$ be a compact connected oriented piecewise linear 4-manifold with an isomorphism $\varphi_{q}$ : $H_{q}(F ; R) \approx H_{q}(W ; R)$ for all $q>0$, where $R=Z$ or $Q$, and such that the intersection number of any two elements of $\mathrm{H}_{2}(W ; Z) /$ (torsions) is 0 . The purpose of this paper is to give some algebraic invariants which are necessary to find a piecewise linear imbedding $F \rightarrow W$ inducing this isomorphism $\varphi_{q}$ for all $q>0$. Such invariants come from some developed arguments of quadratic forms of 3-manifolds defined by the author in [9]. By using these invariants, we shall have some examples.

EXAMPLE 2.5. For each $g \geqslant 1$ there are compact connected orientable 4-manifolds $W$ such that $W$ is homotopy equivalent to a closed connected orientable surface $F_{g}$ of genus $g$, but there is no (possibly nonlocally flat) piecewise linear imbedding from $F_{g}$ to $W$ inducing any homology isomorphism.

For $g=1$ this gives an elementary proof of a spineless 4-manifold announced by Y. Matsumoto [15]. In the higher even dimensional case, S. E. Cappell and J. L. Shaneson [1] and Y. Matsumoto [16] have constructed spineless manifolds $W^{n+2}$ for each even $n \geqslant 4$ whose statements are weaker than the above.

EXAMPLE 2.6. For each $g \geqslant 0$ there are infinitely many relatively nonhomology cobordant compact connected orientable 4-manifolds $W$ such that there is a homotopy equivalent piecewise linear imbedding $F_{g} \rightarrow W$, but there is no locally flat piecewise linear imbedding from $F_{g}$ to $W$ inducing any homology isomorphism.

Let $L$ be a link of $s$ components with linking numbers 0 (i.e. any two components of $L$ have the linking number 0 ) and let $W_{L}$ be a 4-manifold obtained from a 4-cell $D^{4}$ by attaching $s$ 2-handles along the link $L$ in $S^{3}=\partial D^{4}$ with null-homologous framings. Clearly, $W_{L}$ is homotopy equivalent to a bouquet $S^{2} \vee S^{2}$ $\vee \cdots \vee S^{2}$ of $s$ 2-spheres and any two elements of $H_{2}\left(W_{L} ; Z\right)$ have the

Received by the editors November 15, 1978 and, in revised form, October 9, 1979.

AMS (MOS) subject classifications (1970). Primary 57C35; Secondary 55A10, 57C20.

Key words and phrases. 4-manifold, piecewise linear imbedding, quadratic form, Alexander polynomial, local signature.

${ }^{1}$ Research supported in part by a National Science Foundation grant. 
intersection number 0 and every element of $H_{2}\left(W_{L} ; Z\right)$ is represented by a piecewise linearly imbedded 2-sphere.

EXAMPLE 2.7. For each $s \geqslant 2$ there is a boundary link $L$ of $s$ components such that a basis of $\mathrm{H}_{2}\left(W_{L} ; Z\right)$ coming from the components of $L$ cannot be represented by mutually disjoint piecewise linearly imbedded 2-spheres. In particular, for each $s \geqslant 2$ there is a boundary link of $s$ components which is not cobordant to a completely splittable link.

In the higher odd-dimensional case, such a boundary link also exists. (See [6].)

EXAMPLE 2.10. For each $s \geqslant 2$ there is a link $L$ of $s$ components (with linking numbers 0$)$ such that any s elements of $\mathrm{H}_{2}\left(W_{L} ; Z\right)$ forming a basis of $\mathrm{H}_{2}\left(W_{L} ; Q\right)$ cannot be represented by mutually disjoint piecewise linearly imbedded 2-spheres.

In fact, we shall show this for the links $Q_{0}$ and $E_{0}$ of 2-components in Figure 3 considered by $\mathrm{H}$. Lambert [13]. So, neither $Q_{0}$ nor $E_{0}$ bounds two 2-cells mutuallydisjointly and piecewise linearly imbedded in $D^{4}$. This answers a question of $\mathrm{H}$. Lambert [13]. Note that this assertion for $Q_{0}$ and $E_{0}$ is not derived from the arguments of the Robertello-Arf invariants of links. Cf. [13], [17].

EXAMPLE 2.12. For each $s \geqslant 1$ there are compact 4-manifolds $W$ homotopy equivalent to a bouquet of $s$ 2-spheres such that a basis of $\mathrm{H}_{2}(W ; Z)$ is represented by $s$ mutually disjoint piecewise linearly imbedded 2-spheres, but any $s$ elements of $\mathrm{H}_{2}(W ; Z)$ forming a basis of $\mathrm{H}_{2}(W ; Q)$ cannot be represented by mutually disjoint locally flat 2-spheres.

The case $s=1$ is the 4-dimensional result of M. Kato [5, Theorem D].

$\$ 1$ concerns a general argument of quadratic forms of closed oriented odd-dimensional manifolds, where we shall give Fundamental Theorems I and II. In $\$ 2$, we shall give some algebraic invariants for a piecewise linear imbedding $F \rightarrow W$ inducing an isomorphism $\varphi_{q}$ for all $q>0$ and the above examples.

Spaces and maps are considered in the piecewise linear category throughout this paper and it seems that this category is essential for our invariants, because, for example, the Giffen's shift spinning construction produces a homotopy equivalent topological (wild) imbedding $F_{g} \rightarrow W$ for the manifold $W$ in Example 2.5. (See C. H. Giffen [4], W. T. Eaton, C. P. Pixley and G. A. Venema [2] and Y. Matsumoto [18].)

1. Properties of quadratic forms of odd-dimensional manifolds. Let $X$ be a finite complex with $\operatorname{rank}_{Z} H_{1}(X ; Z) \geqslant 1$. Every element of $H^{1}(X ; Z)$ corresponds bijectively to a homomorphism from the free product $F(X)$ of the fundamental groups of the components of $X$ (or $H_{1}(X ; Z)$ ) to a fixed infinite cyclic group $\langle t\rangle$ with a specified generator $t$ by the identification $H^{1}(X ; Z)=\operatorname{Hom}[F(X),\langle t\rangle]$ (or $\left.=\operatorname{Hom}\left[H_{1}(X ; Z),\langle t\rangle\right]\right)$. A nonzero element $\gamma$ of $H^{1}(X ; Z)$ is indivisible if the equation $\gamma=n \gamma^{\prime}$ with $n \in Z$ and $\gamma^{\prime} \in H^{1}(X ; Z)$ implies that $|n|=1$. Indivisible elements of $H^{1}(X ; Z)$ correspond bijectively to epimorphisms from $F(X)$ (or $\left.H_{1}(X ; Z)\right)$ to $\langle t\rangle$. Let $\gamma \in H^{1}(X ; Z)$ be an element and $\tilde{X}$ be the infinite cyclic cover of $X$ associated with $\gamma$, that is, $\tilde{X}$ is the fibered product of a map $f: X \rightarrow S^{1}$ inducing $\gamma$ and the exponential cover exp: $R^{1} \rightarrow S^{1}$ (cf. [7, p. 437]). The covering translation group of $\tilde{X}$ is identified with $\langle t\rangle$ by the evaluation map $\gamma$. For a 
(possibly empty) subcomplex $X^{\prime}$ of $X$, let $\tilde{X}^{\prime}$ be the lift of $X^{\prime}$ by the covering projection $\tilde{X} \rightarrow X$. The rational homology group $H_{*}\left(\tilde{X}, \tilde{X}^{\prime} ; Q\right)=H_{*}\left(\tilde{X}, \tilde{X}^{\prime} ; Q\right)_{\gamma}$ is a finitely generated module over the rational group ring $Q\langle t\rangle$ of $\langle t\rangle . T_{*}\left(\tilde{X}, \tilde{X}^{\prime}\right)$ $=T_{*}\left(\tilde{X}, \tilde{X}^{\prime}\right)_{\gamma}$ is the $Q\langle t\rangle$-torsion part of $H_{*}\left(\tilde{X}, \tilde{X}^{\prime} ; Q\right)$ and $T^{*}\left(\tilde{X}, \tilde{X}^{\prime}\right)=$ $T^{*}\left(\tilde{X}, \tilde{X}^{\prime}\right)_{\gamma}$ is the dual vector space over $Q . T^{*}\left(\tilde{X}, \tilde{X}^{\prime}\right)$ admits a $Q\langle t\rangle$-module structure induced naturally from $T_{*}\left(\tilde{X}, \tilde{X}^{\prime}\right)$ so that $(f u)(x)=u(f x)(f \in Q\langle t\rangle$, $u \in T^{*}\left(\tilde{X}, \tilde{X}^{\prime}\right)$ and $\left.x \in T_{*}\left(\tilde{X}, \tilde{X}^{\prime}\right)\right)$, since $Q\langle t\rangle$ is a commutative ring. The qth Alexander polynomial $A_{q}^{\gamma}(t)$ of the pair $\left(X, X^{\prime}\right)$ with $\gamma \in H^{1}(X ; Z)$ is the order ideal of the $Q\langle t\rangle$-torsion module $T_{q}\left(\tilde{X}, \tilde{X}^{\prime}\right)$ (or equivalently $T^{q}\left(\tilde{X}, \tilde{X}^{\prime}\right)$ ) (cf. [9]). The qth $Q\langle t\rangle$-Betti number $\beta_{q}^{\gamma}\left(X, X^{\prime}\right)$ of the pair $\left(X, X^{\prime}\right)$ with $\gamma \in H^{1}(X ; Z)$ is the $Q\langle t\rangle$-rank of $H_{q}\left(\tilde{X}, \tilde{X}^{\prime} ; Q\right)$. If two nonzero elements $f_{1}(t)$ and $f_{2}(t)$ in $Q\langle t\rangle$ are equal up to units of $Q\langle t\rangle$, then the notation $f_{1}(t) \doteq f_{2}(t)$ is used.

We have the following reduction formula.

LeMma 1.1. Let $\gamma=n \bar{\gamma} \in H^{1}(X ; Z)$ with nonzero $\bar{\gamma} \in H^{1}(X ; Z)$ and nonzero $n \in Z$. Let

$$
T_{q}\left(\tilde{X}, \tilde{X}^{\prime}\right)_{\bar{\gamma}} \approx Q\langle t\rangle /\left(f_{1}(t)\right) \oplus \cdots \oplus Q\langle t\rangle /\left(f_{r}(t)\right)
$$

be a cyclic decomposition. We have that $\beta_{q}^{\gamma}\left(X, X^{\prime}\right)=\beta_{q}^{\bar{\gamma}}\left(X, X^{\prime}\right)$ and

$$
T_{q}\left(\tilde{X}, \tilde{X}^{\prime}\right)_{\gamma} \approx Q\langle t\rangle /\left(f_{1}\left(t^{n}\right)\right) \oplus \cdots \oplus Q\langle t\rangle /\left(f_{r}\left(t^{n}\right)\right)
$$

and, in particular, $A_{q}^{\gamma}(t) \doteq A_{q}^{\bar{\gamma}}\left(t^{n}\right)$.

Proof. Clearly it suffices to prove this lemma for the case that $n \geqslant 2$ and $X$ is connected and $\bar{\gamma}$ is indivisible. [Note that the case that $n=-1$ is obvious and $\gamma=(-n)(-\bar{\gamma})$ for $n \leqslant-2$.] Let $\left(\tilde{X}, \tilde{X}^{\prime}\right)$ be the cover of $\left(X, X^{\prime}\right)$ associated with $\gamma \in H^{1}(X ; Z) . \tilde{X}$ has just $n$ components, since $X$ is connected and $\gamma=n \bar{\gamma}, n \geqslant 2$, and $\bar{\gamma}$ is indivisible. The actions $t, t^{2}, \ldots, t^{n-1}$ are cyclic translations on the components of $\tilde{X}$ and the subgroup $\operatorname{Im} \gamma=\left\langle t^{n}\right\rangle$ of $\langle t\rangle$ acts on each component of $\tilde{X}$. Let $\tilde{X}_{0}$ be any component of $\tilde{X}$. $\tilde{X}_{0}$ is the infinite cyclic cover of $X$ associated with the epimorphism $\gamma_{0}: \pi_{1}(X) \rightarrow \operatorname{Im} \gamma=\left\langle t^{n}\right\rangle$ defined by $\gamma$. Let $\tilde{X}_{0}^{\prime}=\tilde{X}^{\prime} \cap \tilde{X}_{0}$. By assumption we have

$$
H_{q}\left(\tilde{X}_{0}, \tilde{X}_{0}^{\prime} ; Q\right)_{\gamma_{0}} \approx Q\left\langle t^{n}\right\rangle^{\beta} \oplus Q\left\langle t^{n}\right\rangle /\left(f_{1}\left(t^{n}\right)\right) \oplus \cdots \oplus Q\left\langle t^{n}\right\rangle /\left(f_{r}\left(t^{n}\right)\right)
$$

as $Q\left\langle t^{n}\right\rangle$-modules, where $\beta=\beta_{q}^{\bar{\gamma}}\left(X, X^{\prime}\right)$. Hence we obtain that

$$
\begin{aligned}
H_{q}\left(\tilde{X}, \tilde{X}^{\prime} ; Q\right)_{\gamma} & \approx \sum_{i=0}^{n-1} t^{i}\left[Q\left\langle t^{n}\right\rangle^{\beta} \oplus Q\left\langle t^{n}\right\rangle /\left(f_{1}\left(t^{n}\right)\right) \oplus \cdots \oplus Q\left\langle t^{n}\right\rangle /\left(f_{r}\left(t^{n}\right)\right)\right] \\
& \approx Q\langle t\rangle^{\beta} \oplus Q\langle t\rangle /\left(f_{1}\left(t^{n}\right)\right) \oplus \cdots \oplus Q\langle t\rangle /\left(f_{r}\left(t^{n}\right)\right)
\end{aligned}
$$

as $Q\langle t\rangle$-modules. This completes the proof.

Let $M$ be a compact oriented $n$-manifold with $\operatorname{rank}_{Z} H_{1}(M ; Z) \geqslant 1$. Let $\gamma \in H^{1}(M ; Z)$ be a nonzero element and $\tilde{M}$ be the infinite cyclic cover of $M$ associated with $\gamma$. By Duality Theorem (II) and Remark 1.7 of [9], there is a nonsingular pairing

$$
T^{q}(\tilde{M}, \partial \tilde{M}) \times T^{n-q-1}(\tilde{M}) \stackrel{\cup}{\rightarrow} T^{n-1}(\tilde{M}, \partial \tilde{M})
$$


induced from the usual cup product pairing

$$
H^{q}(\tilde{M}, \partial \tilde{M} ; Q) \times H^{n-q-1}(\tilde{M} ; Q) \stackrel{\cup}{\rightarrow} H^{n-1}(\tilde{M}, \partial \tilde{M} ; Q) .
$$

Let $M_{1}, \ldots, M_{c}$ be the components of $M$ such that the restrictions $\gamma \mid M_{i}$ are nonzero, $i=1, \ldots, c$. Let $d=\sum_{i=1}^{c}\left|\langle t\rangle / \operatorname{Im}\left(\gamma \mid M_{i}\right)\right|<+\infty$. Note that $\tilde{M}$ has just $d$ noncompact components and each noncompact component of $\tilde{M}$ is an infinite cyclic cover of some $M_{i}$ associated with an indivisible element $\gamma_{i}$ in $H^{1}\left(M_{i} ; Z\right)$ specified uniquely by the equality $\gamma\left|M_{i}=\right|\langle t\rangle / \operatorname{Im}\left(\gamma \mid M_{i}\right) \mid \gamma_{i}$. Using this infinite cyclic cover, we can have the quasi-fundamental class $^{2}$ for each noncompact component of $\tilde{M}$. It follows that $T_{n-1}(\tilde{M}, \partial \tilde{M})$ is the direct sum of $d$ copies of $Q$ with a basis consisting of the quasi-fundamental classes of the noncompact components of $\tilde{M}$. This basis (as a set) is $t$-invariant and can be specified uniquely by the orientation of $M$ and the element $\gamma \in H^{1}(M ; Z)$. Using the dual basis $u_{1}, \ldots, u_{d}$ of this basis, we define a map $\lambda: T^{n-1}(\tilde{M}, \partial \tilde{M}) \rightarrow Q$ by the equality

$$
\lambda\left(\sum_{i=1}^{d} q_{i} u_{i}\right)=\sum_{i=1}^{d} q_{i} .
$$

In other words, $\lambda$ denotes the Kronecker product of an element $\sum_{i=1}^{d} q_{i} u_{i} \in$ $T^{n-1}(\tilde{M}, \partial \tilde{M})$ with the sum of the quasi-fundamental classes of the noncompact components of $\tilde{M}$. Note that the following triangle

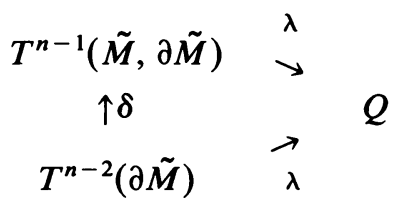

is commutative, since the boundary operator $\partial: T_{n-1}(\tilde{M}, \partial \tilde{M}) \rightarrow T_{n-2}(\partial \tilde{M})$ sends the sum of the quasi-fundamental classes of the noncompact components of $\tilde{M}$ to the sum of the quasi-fundamental classes of the noncompact components of $\partial \tilde{M}$, where $\delta$ is the dual homomorphism of the boundary operator $\partial$. The pairing $T^{q}(\tilde{M}, \partial \tilde{M}) \times T^{n-q-1}(\tilde{M}) \rightarrow Q$ induced by the map $\lambda$ and denoted also by $\cup$, is

${ }^{2}$ Let $M_{*}$ be a compact connected oriented $n$-manifold with an indivisible element $\gamma_{*} \in H^{1}\left(M_{*} ; Z\right)$. Let $\tilde{M}_{*}$ be the infinite cyclic (connected) cover of $M_{*}$ associated with $\gamma_{*}$. The guasi-fundamental class of $\tilde{M}_{*}$ is an element of $T_{n-1}\left(\tilde{M}_{*}, \partial \tilde{M}_{*}\right)(\approx Q)$ which is the image of $1 \in H^{0}\left(M_{*} ; Q\right)$ under a canonical composite monomorphism

$$
H^{0}\left(\tilde{M}_{*} ; Q\right) \stackrel{\delta}{\rightarrow} H_{c}^{1}\left(\tilde{M}_{*} ; Q\right) \stackrel{\bigcap\left[\tilde{M}^{\prime}\right]}{\underset{\approx}{\rightleftarrows}} H_{n-1}\left(\tilde{M}_{*}, \partial \tilde{M}_{*} ; Q\right)
$$

(cf. [7, Definition 2.2] or [9, p. 181]), where note that for a lift $\tilde{f:}_{\tilde{M}_{*}} \rightarrow R^{1}$ of a map $f: M_{*} \rightarrow S^{1}$ representing $\gamma_{*}$ the following square

$$
\begin{array}{ccc}
H^{0}\left(\tilde{M}_{*} ; Q\right) & \stackrel{\delta}{\rightarrow} & H_{c}^{1}\left(\tilde{M}_{*} ; Q\right) \\
\approx \uparrow \tilde{f}^{*} & & \uparrow \tilde{f}^{*} \\
H^{0}\left(R^{1} ; Q\right) & \stackrel{\delta}{\approx} & H_{c}^{1}\left(R^{1} ; Q\right)
\end{array}
$$

is commutative. The quasi-fundamental class of $\tilde{M}_{*}$ is determined uniquely by the orientation of $M_{*}$ and the element $\gamma_{*} \in H^{1}\left(M_{*} ; Z\right)$. 
still nonsingular, because the cup product splits into the components. By [9, Lemma 1.8], and the above remarks, the cup product pairing

$$
H^{q}(\tilde{M}, \partial \tilde{M} ; Q) \times H^{n-q-1}(\tilde{M}, \partial \tilde{M} ; Q) \stackrel{\cup}{\rightarrow} H^{n-1}(\tilde{M}, \partial \tilde{M} ; Q)
$$

induces a pairing (evaluated by the map $\lambda$ )

$$
T^{q}(\tilde{M}, \partial \tilde{M}) \times T^{n-q-1}(\tilde{M}, \partial \tilde{M}) \stackrel{\cup}{\rightarrow} Q
$$

satisfying the identity $x \cup y=j^{*}(x) \cup y=x \cup j^{*}(y)$, where $j^{*}: T^{*}(\tilde{M}, \partial \tilde{M}) \rightarrow$ $T^{*}(\tilde{M})$ is a homomorphism induced by the inclusion $j: \tilde{M} \subset(\tilde{M}, \partial \tilde{M})$.

Let $n=2 m+1(m \geqslant 1)$. A $t$-isometric symmetric bilinear $Q$-form $\langle\rangle=$, $\langle,\rangle_{\gamma}: T^{m}(\tilde{M}, \partial \tilde{M}) \times T^{m}(\tilde{M}, \partial \tilde{M}) \rightarrow Q$ is defined by the identity

for all $x, y \in T^{m}(\tilde{M}, \partial \tilde{M})$.

$$
\langle x, y\rangle= \begin{cases}x \cup\left(t-t^{-1}\right) y & \text { if } m \text { is odd, } \\ x \cup y & \text { if } m \text { is even, }\end{cases}
$$

Definition 1.2. The pair $(\langle\rangle, t$,$) is called the quadratic form of M^{2 m+1}$ with nonzero element $\gamma \in H^{1}(M ; Z)$ (cf. [9, Remark 3.2]).

As an example, let $E(k)$ be the manifold obtained from $S^{3}$ by removing an open tubular neighborhood of an oriented knot $k$ in the oriented 3-sphere $S^{3}$ and $\gamma \in H^{1}(E(k) ; Z)$ be the dual of a generator of $H_{1}(E(k) ; Z)$ linking the knot $k$ in $S^{3}$ by the linking number +1 . The quadratic form

$$
\left(\langle,\rangle_{k}, t\right): T^{1}(\tilde{E}(k), \partial \tilde{E}(k)) \times T^{1}(\tilde{E}(k), \partial \tilde{E}(k)) \rightarrow Q
$$

is necessarily nonsingular and called the quadratic form of the knot $k$ (cf. J. W. Milnor [19]).

For simplicity, we shall henceforth consider a closed $(2 m+1)$-manifold $M^{2 m+1}$. Then in the case that $m$ is even the quadratic form $\langle$,$\rangle is nonsingular by$ definition. In the case that $m$ is odd the (maximal) null subspace $N=N(\tilde{M})$ of this quadratic form $\langle$,$\rangle , whose Q$-dimension is called the nullity of $M$ with $\gamma$ and denoted by $n^{\gamma}(M)$, has a $Q\langle t\rangle$-module structure and is $Q\langle t\rangle$-isomorphic to a direct sum of copies of $Q\langle t\rangle /(t-1)$ and $Q\langle t\rangle /(t+1)$, for $N$ is precisely equal to the kernel of $t-t^{-1}: T^{m}(\tilde{M}) \rightarrow T^{m}(\tilde{M}){ }^{3}$ The signature of the form $\langle$,$\rangle is called$ the signature of $M$ with $\gamma$ and denoted by $\sigma^{\gamma}(M)$. Note that $\sigma^{\gamma}(M)$ for odd $m$ is equal to the signature of the nonsingular form $\langle,\rangle^{\wedge}: \hat{T}^{m}(\tilde{M}) \times \hat{T}^{m}(\tilde{M}) \rightarrow Q$ with $\hat{T}^{m}(\tilde{M})=T^{m}(\tilde{M}) / N(\tilde{M})$ induced from the form $\langle\rangle:, T^{m}(\tilde{M}) \times T^{m}(\tilde{M}) \rightarrow Q$. We now describe the local signature $\sigma_{\omega}^{\gamma}(M)$ at any value $\omega$ in the interval $[-1,1]$ (cf. J. W. Milnor [19]). Consider a nonsingular $t$-isometric symmetric $Q$-form $\langle$,$\rangle :$ $T \times T \rightarrow Q$ with a finitely generated torsion $Q\langle t\rangle$-module $T$. By tensoring with the real field $R$, we consider this form as a nonsingular $t$-isometric symmetric $R$-form $\langle,\rangle_{R}: T_{R} \times T_{R} \rightarrow R . T_{R}$ splits into the $p(t)$-primary components $C_{p(t)}$, where $p(t)$ ranges over all irreducible real polynomials of degree 1 or 2 with the leading coefficient 1 and $C_{p(t)}=0$ except for a finite number of $p(t)$. Because of the

\footnotetext{
${ }^{3}$ Thus, in the case that $m$ is odd, if $A_{m}^{\gamma}( \pm 1) \neq 0$ for the $m$ th Alexander polynomial $A_{m}^{\gamma}(t)$ of $M$ with $\gamma$, then $N=0$, i.e., the quadratic form $\langle$,$\rangle is nonsingular.$
} 
equality $\langle f(t) x, y\rangle_{R}=\left\langle x, f\left(t^{-1}\right) y\right\rangle_{R}$, the signature of the form $\langle,\rangle_{R}$ (and hence of $\langle\rangle$,$) is the sum of the signatures of the forms restricted to C_{p(t)}$ with $p(t)=t-1, t+1$ or $t^{2}-2 \omega t+1,-1<\omega<1$ (cf. J. W. Milnor [19, p. 129]). [Note that if $p_{1}(t) \neq r t^{a} p_{2}\left(t^{-1}\right), r \in R$, then $C_{p_{i}(t)}, i=1,2$, are orthogonal, i.e., $C_{p_{1}(t)} \perp C_{p_{2}(t)}$, and if $p_{1}(t)=r t^{a} p_{2}\left(t^{-1}\right), r \in R$, but $p_{1}(t) \neq p_{2}(t)$, then $C_{p_{i}(t)} \perp C_{p_{i}(t)}$, $i=1$, 2. (See J. W. Milnor [20, Lemma 3.1].) Further, if $p(t)=r t^{a} p\left(t^{-1}\right), r \in R$, then $p(t)$ must be $t-1, t+1$ or $t^{2}-2 \omega t+1,-1<\omega<1$.]

Definition 1.3 (J. W. Milnor [19]). For each value $\omega,-1 \leqslant \omega \leqslant 1$, $\sigma_{\omega}^{\gamma}$ is the signature of the restricted form of $\langle,\rangle_{R}$ to the component $C_{p(t)}$, where $p(t)=t-$ $1, t+1$ or $t^{2}-2 \omega t+1$ according as $\omega=1,-1$ or $-1<\omega<1$, and called the local signature of the nonsingular form $\langle$,$\rangle at \omega \in[-1,1]$. The local signature of the nonsingular form $\langle,\rangle^{\wedge}: \hat{T}^{m}(\tilde{M}) \times \hat{T}^{m}(\tilde{M}) \rightarrow Q$ for odd $m$ or $\langle\rangle:, T^{m}(\tilde{M}) \times$ $T^{m}(\tilde{M}) \rightarrow Q$ for even $m$ at $\omega \in[-1,1]$ is called the local signature of $M$ with $\gamma \in H^{1}(M ; Z)$ at $\omega \in[-1,1]$ and denoted by $\sigma_{\omega}^{\gamma}(M)$.

LEMMA 1.4 (J. W. MILNOR). The signature of a t-isometric symmetric nonsingular $R$-form of the following type

$$
\langle,\rangle_{R}: R\langle t\rangle /\left(t^{2}-2 \omega t+1\right) \times R\langle t\rangle /\left(t^{2}-2 \omega t+1\right) \rightarrow R, \quad-1<\omega<1,
$$

is \pm 2 (cf. [19, Assertion 11]).

Definition 1.5 (J. LeVINE [14]). The nonsingular $t$-isometric symmetric $Q$-form $(\langle\rangle, t):, T \times T \rightarrow Q$ with a finitely generated torsion $Q\langle t\rangle$-module $T$ is nullcobordant, if there is a $Q\langle t\rangle$-submodule $T_{0}$ of $T$ that is a self-orthogonal complement, i.e., $T_{0}^{\perp}=T_{0}$ with respect to this form $\langle$,$\rangle . Two nonsingular t$-isometric symmetric forms $\left(\langle,\rangle^{i}, t\right), i=1,2$, are cobordant, if the orthogonal sum $\left(\langle,\rangle^{1} \perp-\langle,\rangle^{2}, t\right)$ is null-cobordant.

Clearly the signatures of cobordant forms are equal. We also have the following

LEMMA 1.6. The local signatures of cobordant forms at any $\omega \in[-1,1]$ are equal.

Proof. It suffices to show that the local signature at any $\omega \in[-1,1]$ of a null-cobordant form is 0 . Consider a null-cobordant form $\left(\langle,\rangle_{R}, t\right): T_{R} \times T_{R} \rightarrow R$ over $R$ with $T_{R}^{0 \perp}=T_{R}^{0}$. Let $p(t)=t-1, t+1$ or $t^{2}-2 \omega t+1,-1<\omega<1$. Note that the restricted form of $\langle,\rangle_{R}$ to $C_{p(t)}$ is nonsingular, since for any different $q(t), C_{p(t)} \perp C_{q(t)}$. Let $y \in C_{p(t)}$. Suppose $\langle x, y\rangle=0$ for all $x \in T_{R}^{0} \cap C_{p(t)}$. Using that $C_{p(t)} \perp C_{q(t)}$ for a different $q(t)$, we obtain that $\langle x, y\rangle=0$ for all $x \in T_{R}^{0}$ and hence $y \in T_{R}^{0} \cap C_{p(t)}$. This implies that $\left(T_{R}^{0} \cap C_{p(t)}\right)^{\perp}=T_{R}^{0} \cap C_{p(t)}$. Now the conclusion easily follows. This completes the proof.

Notations 1.7. Let $\tilde{X}$ be an infinite cyclic cover of a finite complex $X$. The $Q\langle t\rangle$-torsion module $T_{*}(\tilde{X})$ splits into the $p(t)$-primary components over $Q\langle t\rangle$, where $p(t)$ is a primitive, ${ }^{4}$ nonconstant, irreducible polynomial. The $p(t)$-primary component of $T_{*}(\tilde{X})$ with $p(t)=t-1$ or $t+1$ is denoted by ${ }_{1} U_{*}(\tilde{X})$ or ${ }_{-1} U_{*}(\tilde{X})$, respectively, and the direct summand consisting of all the $p(t)$-primary components with $|p(1)| \neq 1$ is denoted by $U_{*}(\tilde{X})$. The corresponding parts of

\footnotetext{
${ }^{4}$ A nonzero element of $Q\langle t\rangle$ is primitive, if it is written as either the constant 1 or an integral polynomial $c_{n} t^{n}+c_{n-1} t^{n-1}+\cdots+c_{0}(n>0)$ with $c_{n}(>0), c_{n-1}, \ldots, c_{0}(\neq 0)$ coprime (cf. [9]).
} 
$T^{*}(\tilde{X})$ to ${ }_{i} U_{*}(\tilde{X})$ and $U_{*}(\tilde{X})$ are denoted by ${ }_{i} U^{*}(\tilde{X})$ and $U^{*}(\tilde{X})$, respectively. Similar notations are used for a pair $\left(\tilde{X}, \tilde{X}^{\prime}\right)$.

The following Fundamental Theorem $I$ is a generalization of Theorem 4.3 in [9] which is a main tool to show a failure of the 4-dimensional Whitney lemma in the piecewise linear category (cf. [9, Theorem C]).

Fundamental TheOREM I. Let $X_{i}, i=1,2$, be finite complexes with nonzero elements $\gamma_{i} \in H^{1}\left(X_{i} ; Z\right)$ imbedded in a finite complex $Y$ so that $\gamma_{i}$ are the restrictions on the same element $\gamma \in H^{1}(Y ; Z)$ to $X_{i}$. If $H_{q}\left(Y, X_{i} ; Q\right)=H_{q+1}\left(Y, X_{i} ; Q\right)=0$, $i=1,2$, then $\beta_{q}^{\gamma_{1}}\left(X_{1}\right)=\beta_{q}^{\gamma_{2}}\left(X_{2}\right)$ and ${ }_{1} U_{q}\left(\tilde{X}_{1}\right)$ is $Q\langle t\rangle$-isomorphic to ${ }_{1} U_{q}\left(\tilde{X}_{2}\right)$ and so the multiplicities of $t=1$ in the roots of the qth Alexander polynomials $A_{q}^{\gamma_{i}}(t)$ are equal. Moreover, if $H_{q}\left(Y, X_{i} ; Z\right)=H_{q+1}\left(Y, X_{i} ; Z\right)=0$, then $U_{q}\left(\tilde{X}_{1}\right)$ is $Q\langle t\rangle$-isomorphic to $U_{q}\left(\tilde{X}_{2}\right)$ and, in particular, $A_{q}^{\gamma_{1}}(t) \doteq A_{q}^{\gamma_{2}}(t)$ (up to $f(t)$ with $f(t) \in Z\langle t\rangle$ and $|f(1)|=1)$.

REMARK 1.8. A version using a finite field $Z_{p}$ instead of $Q$ is also possible.

The proof of Fundamental Theorem $I$ is parallel to the proof of Theorem 4.3 in [9]. We sketch here only an outline of the proof. It proceeds as follows:

$$
H_{j}\left(Y, X_{i} ; Q\right)=0
$$

implies that $H_{j}\left(\tilde{Y}, \tilde{X}_{i} ; Q\right)=T_{j}\left(\tilde{Y}, \tilde{X}_{i}\right)$ and ${ }_{1} U_{j}\left(\tilde{Y}, \tilde{X}_{i}\right)=0$. [Use the Wang exact sequence.] From the exact sequence of the pair $\left(\tilde{Y}, \tilde{X}_{i}\right)$, it follows that $\beta_{q}^{\gamma_{i}}\left(X_{i}\right)=$ $\beta_{q}^{\gamma}(Y)$ and the sequence

$$
T_{q+1}\left(\tilde{Y}, \tilde{X}_{i}\right) \stackrel{\partial}{\rightarrow} T_{q}\left(\tilde{X}_{i}\right) \stackrel{i_{*}}{\rightarrow} T_{q}(\tilde{Y}) \stackrel{j_{*}}{\rightarrow} T_{q}\left(\tilde{Y}, \tilde{X}_{i}\right)
$$

is exact and so ${ }_{1} U_{q}\left(\tilde{X}_{i}\right)$ is $Q\langle t\rangle$-isomorphic to ${ }_{1} U_{q}(\tilde{Y})(i=1,2)$. If

$$
H_{j}\left(Y, X_{i} ; Z\right)=0 \text {, }
$$

then we have $U_{j}\left(\tilde{Y}, \tilde{X}_{i}\right)=0$. Hence the above exact sequence implies that $U_{q}\left(\tilde{X}_{i}\right)$ is $Q\langle t\rangle$-isomorphic to $U_{q}(\tilde{Y})$. In particular, $A_{q}^{\gamma_{1}}(t) f_{1}(t) \doteq A_{q}^{\gamma_{2}}(t) f_{2}(t)$ for some $f_{i}(t) \in$ $Z\langle t\rangle$ with $\left|f_{i}(1)\right|=1$. This completes the outlined proof of Fundamental Theorem I.

Fundamental THeOREM II. Let $M$ be the boundary of a compact oriented $(2 m+2)$-manifold $W$ with a nonzero element $\bar{\gamma} \in H^{1}(W ; Z)$ extending a nonzero element $\gamma \in H^{1}(M ; Z)$. Suppose that the sequence

$$
T^{m}(\tilde{W}) \stackrel{i^{*}}{\rightarrow} T^{m}(\tilde{M}) \stackrel{\delta}{\rightarrow} T^{m+1}(\tilde{W}, \tilde{M})
$$

is exact at $T^{m}(\tilde{M})$. Then for even $m$ the quadratic form $(\langle\rangle, t):, T^{m}(\tilde{M}) \times T^{m}(\tilde{M})$ $\rightarrow Q$ is null-cobordant. In particular, $A_{m}^{\gamma}(t) \doteq f(t) f\left(t^{-1}\right)$ for some $f(t) \in Q\langle t\rangle$ and $\sigma_{\omega}^{\gamma}(M)=0$ at all values $\omega \in[-1,1]$ and $\sigma^{\gamma}(M)=0$. For odd $m A_{m}^{\gamma}(t) \doteq f(t) f\left(t^{-1}\right)$ for some $f(t) \in Q\langle t\rangle$ and $\sigma_{\omega}^{\gamma}(M)=0$ at all values $\omega \in(-1,1)$. Further, for odd $m$ if the kernel of $t-t^{-1}: \operatorname{Im} \delta \rightarrow \operatorname{Im} \delta$ is equal to the image of $N(\tilde{M})$ by the map $\delta$, then $N(\tilde{M})$ is $Q\langle t\rangle$-isomorphic to the direct double $\delta(N(\tilde{M})) \oplus \delta(N(\tilde{M}))$ and, in particular, $n^{\gamma}(M) \equiv 0(\bmod 2)$ and the induced nonsingular form $(\langle\rangle, t):, \hat{T}^{m}(\tilde{M}) \times$ $\hat{T}^{m}(\tilde{M}) \rightarrow Q$ is null-cobordant. In particular, $\sigma_{\omega}^{\gamma}(M)=0$ at all values $\omega \in[-1,1]$ and $\sigma^{\gamma}(M)=0$. 
1.9. Proof of Fundamental Theorem II. Given $y \in T^{m}(\tilde{M})$, suppose $i^{*}(x) \cup y$ $=0$ for all $x \in T^{m}(\tilde{M})$. By [9, Lemma 1.8], $x \cup \delta(y)=0$ for all $x$. Hence $\delta(y)=0$, for the cup product pairing $T^{m}(\tilde{W}) \times T^{m+1}(\tilde{W}, \tilde{M}) \stackrel{\cup}{\rightarrow} Q$ is nonsingular ([9, Duality Theorem (II)]). Using the exact sequence

$$
T^{m}(\tilde{W}) \stackrel{i^{*}}{\rightarrow} T^{m}(\tilde{M}) \stackrel{\delta}{\rightarrow} T^{m+1}(\tilde{W}, \tilde{M})
$$

we see that $y$ lies in $\operatorname{Im} i^{*}$. It follows that $\operatorname{Im} i^{*}$ is self-orthogonal, i.e., $\left(\operatorname{Im} i^{*}\right)^{\perp}=$ $\operatorname{Im} i^{*}$ concerning the nonsingular cup product pairing $T^{m}(\tilde{M}) \times T^{m}(\tilde{M}) \stackrel{\cup}{\rightarrow} Q$. Since the sequence $0 \rightarrow \operatorname{Im} i^{*} \rightarrow T^{m}(\tilde{M}) \stackrel{\delta}{\rightarrow} \operatorname{Im} \delta \rightarrow 0$ is exact, from this fact and the equality $(t x) \cup y=x \cup\left(t^{-1} y\right)$ we obtain that $A_{m}^{\gamma}(t) \doteq f(t) f\left(t^{-1}\right)$, where $f(t)$ is the order ideal of $\operatorname{Im} i^{*}$. For even $m$, we also obtain that the form $(\langle\rangle, t$,$) :$ $T^{m}(\tilde{M}) \times T^{m}(\tilde{M}) \rightarrow Q$ is null-cobordant and in particular $\sigma_{\omega}^{\gamma}(M)=0$ at all $\omega \in$ $[-1,1]$ and $\sigma^{\gamma}(M)=0$. Let $m$ be odd. Let $T$ be the direct summand of $T^{m}(\tilde{M})$ consisting of the $p(t)$-primary components such that $p( \pm 1) \neq 0$. The quadratic form $(\langle\rangle, t):, T^{m}(\tilde{M}) \times T^{m}(\tilde{M}) \rightarrow Q$ then restricts to the nonsingular form $(\langle\rangle, t):, T \times T \rightarrow Q$, since the order ideal $P(t)$ of $T$ satisfies $P( \pm 1) \neq 0$. [Note that this asserts an isomorphism $t-t^{-1}: T \approx T$.]

We have $\left(T \cap \operatorname{Im} i^{*}\right)^{\perp}=T \cap \operatorname{Im} i^{*}$ concerning this nonsingular pairing. By Lemma 1.6 this implies that $\sigma_{\omega}^{\gamma}(M)=0$ at $\omega \in(-1,1)$.

Assume that the kernel of $t-t^{-1}: \operatorname{Im} \delta \rightarrow \operatorname{Im} \delta$ is equal to $\delta(N(\tilde{M}))$. Then we have that the kernel sequence of the following morphism

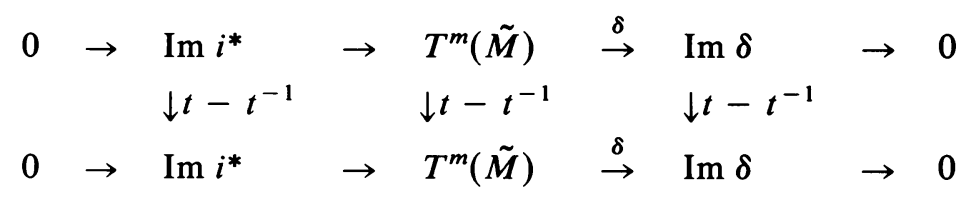

is exact. Dividing by this kernel exact sequence, we obtain the quotient exact sequence $0 \rightarrow \operatorname{Im} i^{\hat{*}} \rightarrow \hat{T}^{m}(\tilde{M}) \stackrel{\delta}{\rightarrow} \operatorname{Im} \hat{\delta} \rightarrow 0$. Now it is easy to see that $\left(\operatorname{Im} i^{*}\right)^{\perp}=$ $\operatorname{Im} i^{\hat{*}}$ concerning the nonsingular form $\langle,\rangle^{\wedge}: \hat{T}^{m}(\tilde{M}) \times \hat{T}^{m}(\tilde{M}) \rightarrow Q$. Therefore the form $\left(\langle,\rangle^{\wedge}, t\right)$ is null-cobordant and $\sigma_{\omega}^{\gamma}(M)=0$ at all $\omega \in[-1,1]$ and $\sigma^{\gamma}(M)=0$. Since the induced pairing $\operatorname{Im} i^{*} \times T^{m}(\tilde{M}) / \operatorname{Im} i^{*} \stackrel{\cup}{\rightarrow} Q$ is nonsingular and $T^{m}(\tilde{M}) / \operatorname{Im} i^{*} \approx \operatorname{Im} \delta$, the kernel of $t-t^{-1}: \operatorname{Im} i^{*} \rightarrow \operatorname{Im} i^{*}$ is $Q\langle t\rangle$-isomorphic to the kernel of $t-t^{-1}: \operatorname{Im} \delta \rightarrow \operatorname{Im} \delta$ that is $\delta(N(\tilde{M}))$ by assumption. Hence $N(\tilde{M})$ is $Q\langle t\rangle$-isomorphic to the duplication $\delta(N(\tilde{M})) \oplus \delta(N(\tilde{M}))$, since $N(\tilde{M})$ is $Q\langle t\rangle$-isomorphic to a direct sum of some copies of $Q\langle t\rangle /(t-1)$ and $Q\langle t\rangle /(t+1)$. In particular, $n^{\gamma}(M) \equiv 0 \bmod 2$. This completes the proof.

DEFINITION 1.10. Two closed connected oriented $n$-manifolds $M_{i}, i=1,2$, with nonzero elements $\gamma_{i} \in H^{1}\left(M_{i} ; Z\right)$ are homology cobordant (or rationally homology cobordant, respectively), if there is a compact connected oriented cobordism $W^{n+1}$ with $\partial W=M_{1} \cup-M_{2}$ such that $H_{*}\left(W, M_{i} ; Z\right)=0\left(\right.$ or $H_{*}\left(W, M_{i} ; Q\right)=0$, respectively) and $\gamma_{i}$ are the restrictions of the same element $\gamma \in H^{1}(W ; Z)$ to $M_{i}$.

We have two standard consequences of Fundamental Theorems I and II. 
Corollary 1.11. Suppose $\left(M_{1}^{2 m+1}, \gamma_{1}\right)$ and $\left(M_{2}^{2 m+1}, \gamma_{2}\right)$ are homology cobordant. Then

(a) $\beta_{*}^{\gamma_{1}}\left(M_{1}\right)=\beta_{*}^{\gamma_{2}}\left(M_{2}\right)$

(b) $U_{*}\left(\tilde{M}_{1}\right) \approx U_{*}\left(\tilde{M}_{2}\right), N\left(\tilde{M}_{1}\right) \approx N\left(\tilde{M}_{2}\right)$ and $n^{\gamma_{1}}\left(M_{1}\right)=n^{\gamma_{2}}\left(M_{2}\right)$,

(c) $A_{q}^{\gamma_{1}}(t) \doteq A_{q}^{\gamma_{2}}(t)$ (up to $f(t)$ with $f(t) \in Z\langle t\rangle,|f(1)|=1$ if $q \neq m$ or up to $f(t) f\left(t^{-1}\right)$ with $f(t) \in Z\langle t\rangle,|f(1)|=1$ if $\left.q=m\right)$,

(d) for even $m$ the quadratic forms $\left.(<,\rangle_{i}, t\right): T^{m}\left(\tilde{M}_{i}\right) \times T^{m}\left(\tilde{M}_{i}\right) \rightarrow Q$ are cobordant and for odd $m$ the induced nonsingular forms $\left(\langle,\rangle_{i}, t\right): \hat{T}^{m}\left(\tilde{M}_{i}\right) \times$ $\hat{T}^{m}\left(\tilde{M}_{i}\right) \rightarrow Q$ are cobordant, $i=1,2$,

(e) $\sigma_{\omega}^{\gamma_{1}}\left(M_{1}\right)=\sigma_{\omega}^{\gamma_{2}}\left(M_{2}\right)$ at all $\omega \in[-1,1]$ and $\sigma^{\gamma_{1}}\left(M_{1}\right)=\sigma^{\gamma_{2}}\left(M_{2}\right)$.

Proof. Note that $T_{m+1}(\tilde{W}, \partial \tilde{W}) \stackrel{\partial}{\rightarrow} T_{m}(\partial \tilde{W}) \stackrel{i_{*}}{\rightarrow} T_{m}(\tilde{W})$ is exact, since the canonical homomorphism $j_{*}: H_{m+1}(\tilde{W} ; Q) \rightarrow H_{m+1}(\tilde{W}, \partial \tilde{W} ; Q)$ is the composite $H_{m+1}(\tilde{W} ; Q) \rightarrow H_{m+1}\left(\tilde{W}, \tilde{M}_{i} ; Q\right) \rightarrow H_{m+1}(\tilde{W}, \partial \tilde{W} ; Q)$ and $T_{m+1}\left(\tilde{W}, \tilde{M}_{i}\right)=$ $H_{m+1}\left(\tilde{W}, \tilde{M}_{i} ; Q\right)$. Hence the dual sequence $T^{m}(\tilde{W}) \stackrel{i^{*}}{\rightarrow} T^{m}(\partial \tilde{W}) \stackrel{\delta}{\rightarrow} T^{m+1}(\tilde{W}, \partial \tilde{W})$ is exact. Then (a), (b), (c), (d) ( $m=$ even) and (e) $(m=$ even) follow from Fundamental Theorems I and II. [Note that the order ideal of $T^{m}(\partial \tilde{W})$ is the

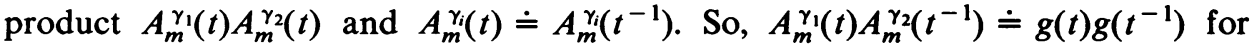
some $g(t) \in Q\langle t\rangle$ and hence $A_{m}^{\gamma_{1}}(t) g_{1}(t) g_{1}\left(t^{-1}\right) \doteq A_{m}^{\gamma_{2}}(t) g_{2}(t) g_{2}\left(t^{-1}\right)$ for some $g_{i}(t)$ $\in Z\langle t\rangle$ with $\left|g_{i}(1)\right|=1$, since $A_{m}^{\gamma_{1}}(t) \doteq A_{m}^{\gamma_{2}}(t)$ up to $f(t) \in Z\langle t\rangle$ with $|f(1)|=1$. Further, note that $\langle\rangle=,\langle,\rangle_{1} \perp-\langle,\rangle_{2}$ for the form $\langle\rangle:, T^{m}(\partial \tilde{W}) \times T^{m}(\partial \tilde{W})$ $\rightarrow Q$.] Next, note that the above exact sequence induces a short exact sequence

$$
0 \rightarrow U^{m}(\tilde{W}) \stackrel{i^{* \prime}}{\rightarrow} U^{m}(\partial \tilde{W}) \stackrel{\delta^{\prime}}{\rightarrow} U^{m+1}(\tilde{W}, \partial \tilde{W}) \rightarrow 0
$$

by using $U^{m}(\partial \tilde{W}) \approx U^{m}\left(\tilde{M}_{1}\right) \oplus U^{m}\left(\tilde{M}_{1}\right)$ (by (b)) and $U^{m}(\tilde{W}) \approx U^{m}\left(\tilde{M}_{1}\right)$ and (by [9, Duality Theorem (II)]) $U^{m+1}(\tilde{W}, \partial \tilde{W}) \approx U^{m}(\tilde{W}) \approx U^{m}\left(\tilde{M}_{1}\right)$. Noting these isomorphisms again, this short exact sequence also induces a short exact sequence

$$
0 \rightarrow N^{m}(\tilde{W}) \stackrel{i^{* \prime \prime}}{\rightarrow} N(\partial \tilde{W}) \stackrel{\delta^{\prime \prime}}{\rightarrow} N^{m+1}(\tilde{W}, \partial \tilde{W}) \rightarrow 0
$$

where $N^{m}(\tilde{W})$ and $N^{m+1}(\tilde{W}, \partial \tilde{W})$ are the kernels of $t-t^{-1}: U^{m}(\tilde{W}) \rightarrow U^{m}(\tilde{W})$ and $t-t^{-1}: U^{m+1}(\tilde{W}, \partial \tilde{W}) \rightarrow U^{m+1}(\tilde{W}, \partial \tilde{W})$, respectively. This implies that the kernel of $t-t^{-1}: \operatorname{Im} \delta \rightarrow \operatorname{Im} \delta$ is precisely equal to $N^{m+1}(\tilde{W}, \partial \tilde{W})=\delta(N(\partial \tilde{W}))$. Therefore by Fundamental Theorem II the induced nonsingular form $(\langle,\rangle \wedge, t)$ : $\hat{T}^{m}(\partial \tilde{W}) \times \hat{T}^{m}(\partial \tilde{W}) \rightarrow Q$ for odd $m$ is null-cobordant. But $\langle,\rangle^{\wedge}=\langle,\rangle_{\hat{i}} \perp$ $-\langle,\rangle_{\hat{2}}$. This shows (d) $(m=$ odd $)$. From Lemma 1.6 , (e) $(m=$ odd $)$ also follows. This completes the proof.

REMARK 1.12. The property (c) can be also generalized to the many-variable Alexander polynomials (cf. [10]).

EXAmple 1.13. Let $D_{1} \cup D_{2} \cup \cdots \cup D_{n}$ be the disjoint union of 2-cells properly and piecewise linearly and locally-flatly imbedded in a 4-cell $D^{4}$. Let $M$ be the boundary of the 4-manifold obtained from $D^{4}$ by removing a regular neighborhood of $D_{1} \cup \cdots \cup D_{n}$ in $D^{4}$ meeting $\partial D^{4}$ regularly. Clearly, $M$ with an arbitrary nonzero $\gamma \in H^{1}(M ; Z)$ is homology cobordant to the connected sum $\#{ }^{n} S^{1} \times S^{2}$ 
with some $\gamma^{\prime} \in H^{1}\left(\#^{n} S^{1} \times S^{2} ; Z\right)$. By Corollary 1.11, we have that $\beta_{1}^{\gamma}(M)=n$ $-1, n^{\gamma}(M)=0, A_{1}^{\gamma}(t) \doteq f(t) f\left(t^{-1}\right), f(t) \in Z\langle t\rangle,|f(1)|=1$, and $\sigma^{\gamma}(M)=\sigma_{\omega}^{\gamma}(M)$ $=0$, all $\omega \in[-1,1]$, and the quadratic form $(\langle\rangle, t):, T^{1}(\tilde{M}) \times T^{1}(\tilde{M}) \rightarrow Q$ is nonsingular and null-cobordant for all nonzero $\gamma \in H^{1}(M ; Z)$ (cf. K. Murasugi [21], A. Kawauchi [9], [10]).

COROLlaRY 1.14. Suppose $\left(M_{1}^{2 m+1}, \gamma_{1}\right)$ and $\left(M_{2}^{2 m+1}, \gamma_{2}\right)$ are rationally homology cobordant. Then

(a) $\beta_{*}^{\gamma_{1}}\left(M_{1}\right)=\beta_{*}^{\gamma_{2}}\left(M_{2}\right)$,

(b) ${ }_{1} U_{*}\left(\tilde{M}_{1}\right) \approx_{1} U_{*}\left(\tilde{M}_{2}\right)$,

(c) $A_{q}^{\gamma_{1}}(t) \doteq A_{q}^{\gamma_{2}}(t)$ (up to $f(t) \in Q\langle t\rangle$ with $f(1) \neq 0$ if $q \neq m$ or up to $f(t) f\left(t^{-1}\right)$ with $f(t) \in Q\langle t\rangle$ and $f(1) \neq 0$ if $q=m$ ),

(d) if $m$ is even, then the quadratic forms $\left(\langle,\rangle_{i}, t\right): T^{m}\left(\tilde{M}_{i}\right) \times T^{m}\left(\tilde{M}_{i}\right) \rightarrow Q$, $i=1,2$, are cobordant and, in particular, $\sigma_{\omega}^{\gamma_{1}}\left(M_{1}\right)=\sigma_{\omega}^{\gamma_{2}}\left(M_{2}\right)$ at all $\omega \in[-1,1]$ and $\sigma^{\gamma_{1}}\left(M_{1}\right)=\sigma^{\gamma_{2}}\left(M_{2}\right)$,

(e) if $m$ is odd, then $\sigma_{\omega}^{\gamma_{1}}\left(M_{1}\right)=\sigma_{\omega}^{\gamma_{2}}\left(M_{2}\right)$ at all $\omega \in(-1,1]$.

Moreover, for odd $m$ assume that ${ }_{-1} U^{m}\left(\tilde{M}_{i}\right) \subset N\left(\tilde{M}_{i}\right), i=1,2$. Then

(f) $N\left(\tilde{M}_{1}\right) \oplus N\left(\tilde{M}_{2}\right)$ is $Q\langle t\rangle$-isomorphic to a direct double $N^{\prime} \oplus N^{\prime}$ and $n^{\gamma_{1}}\left(M_{1}\right)$ $\equiv n^{\gamma_{2}}\left(M_{2}\right)(\bmod 2)$

(g) the induced forms $\left(\langle,\rangle_{i}, t\right): \hat{T}^{m}\left(\tilde{M}_{i}\right) \times \hat{T}^{m}\left(\tilde{M}_{i}\right) \rightarrow Q, i=1,2$, are cobordant,

(h) $\sigma^{\gamma_{1}}\left(M_{1}\right)=\sigma^{\gamma_{2}}\left(M_{2}\right)$.

Proof. By an analogous method of Corollary 1.11, we obtain (a), (b), (c) and (d). The sequence $0 \rightarrow \operatorname{Im} i^{*} \rightarrow T^{m}(\partial \tilde{W}) \stackrel{\delta}{\rightarrow} \operatorname{Im} \delta \rightarrow 0$ and the induced sequence 0 $\rightarrow_{1} U^{m}(\tilde{W}) \stackrel{i^{* \prime}}{\rightarrow} U_{1}^{m}(\partial \tilde{W}) \stackrel{\delta^{\prime}}{\rightarrow}{ }_{1} U^{m+1}(\tilde{W}, \partial \tilde{W}) \rightarrow 0$ are exact, and the kernel sequence of the following morphism is also exact.

$$
\begin{aligned}
& 0 \rightarrow{ }_{1} U^{m}(\tilde{W}) \stackrel{i^{* \prime}}{\rightarrow} \quad{ }_{1} U^{m}(\partial \tilde{W}) \stackrel{\delta^{\prime}}{\rightarrow} \quad{ }_{1} U^{m+1}(\tilde{W}, \partial \tilde{W}) \rightarrow 0 \\
& \downarrow t-t^{-1} \quad \downarrow t-t^{-1} \quad \downarrow t-t^{-1} \\
& 0 \rightarrow{ }_{1} U^{m}(\tilde{W}) \stackrel{i^{* \prime}}{\rightarrow} \quad{ }_{1} U^{m}(\partial \tilde{W}) \stackrel{\delta^{\prime}}{\rightarrow} \quad{ }_{1} U^{m+1}(\tilde{W}, \partial \tilde{W}) \quad \rightarrow \quad 0
\end{aligned}
$$

Thus, $\sigma_{\omega}^{\gamma_{1}}\left(M_{1}\right)-\sigma_{\omega}^{\gamma_{2}}\left(M_{2}\right)=0$ at all $\omega \in(-1,1]$ for odd $m$, showing (e). Let $m$ be odd. If ${ }_{-1} U^{m}\left(\tilde{M}_{i}\right) \subset N\left(\tilde{M}_{i}\right)(i=1,2)$, then combining this with the above remarks we obtain that the kernel of $t-t^{-1}: \operatorname{Im} \delta \rightarrow \operatorname{Im} \delta$ is equal to $\delta(N(\partial \tilde{W})$ ). Therefore, by Fundamental Theorem II we have (f), (g) and (h). This completes the proof.

The above two corollaries will be positively used in the next section and an intended paper [12].

2. A piecewise linear imbedding of a surface into some 4-manifold. Let $F$ be a closed oriented surface with $s(\geqslant 1)$ components $F_{1}, \ldots, F_{s}$ of genera $g_{1}, \ldots, g_{s}$. Consider a compact connected oriented piecewise linear 4-manifold $W$ with an isomorphism $\varphi_{q}: H_{q}(F ; Z) \approx H_{q}(W ; Z)$ for all $q>0$ and such that the intersection number of any two elements of $H_{2}(W ; Z)$ is 0 . It follows that $\partial W$ is connected and the sequence

$$
0 \rightarrow H_{2}(W, \partial W ; Z) \stackrel{\partial}{\rightarrow} H_{1}(\partial W ; Z) \stackrel{i_{*}}{\rightarrow} H_{1}(W ; Z) \rightarrow 0
$$


is exact. Let $\varphi_{2}\left[F_{1}\right]^{*}, \ldots, \varphi_{2}\left[F_{s}\right]^{*}$ be the basis of $H^{2}(W ; Z)$ dual to the basis $\varphi_{2}\left[F_{1}\right], \ldots, \varphi_{2}\left[F_{s}\right]$ of $H_{2}(W ; Z)$, where $\left[F_{i}\right]$ is the fundamental class of $F_{i}$. Let $a_{1}, \ldots, a_{s}$ be the elements of $H_{1}(\partial W ; Z)$ obtained from $\varphi_{2}\left[F_{1}\right]^{*}, \ldots, \varphi_{2}\left[F_{s}\right]^{*}$ by the composite monomorphism

$$
H^{2}(W ; Z) \stackrel{\cap[W]}{\approx} H_{2}(W, \partial W ; Z) \stackrel{\partial}{\rightarrow} H_{1}(\partial W ; Z) .
$$

Next, let $\varphi_{1} H_{1}\left(F_{1} ; Z\right)^{*}, \ldots, \varphi_{1} H_{1}\left(F_{s} ; Z\right)^{*}$ be the direct summands of $H^{1}(W ; Z)$ dual to $\varphi_{1} H_{1}\left(F_{1} ; Z\right), \ldots, \varphi_{1} H_{1}\left(F_{s} ; Z\right)$ by using the decomposition $H_{1}(W ; Z)=\varphi_{1} H_{1}\left(F_{1} ; Z\right) \oplus \cdots \oplus \varphi_{1} H_{1}\left(F_{s} ; Z\right)$. Let $G_{1}, \ldots, G_{s}$ be the subgroups of $H^{1}(\partial W ; Z)$ obtained from $\varphi_{1} H_{1}\left(F_{1} ; Z\right)^{*}, \ldots, \varphi_{1} H_{1}\left(F_{s} ; Z\right)^{*}$ by the canonical monomorphism $i^{*}: H^{1}(W ; Z) \rightarrow H^{1}(\partial W ; Z)$.

Definition 2.1. Let $H$ be an abelian group and let $H^{*}=\operatorname{Hom}[H,\langle t\rangle]$ and $G$ be a subgroup of $H^{*}$. An element $\gamma$ of $H^{*}$ is independent of $G$, if $\gamma(x)=1$ for $x \in H$ such that $\gamma^{\prime}(x) \neq 1$ for some $\gamma^{\prime} \in G$.

Suppose a piecewise linear imbedding $\tilde{\varphi}: F \rightarrow W$ inducing this isomorphism $\varphi_{q}$, $q>0$. Then $\tilde{\varphi}$ is homotopic to a piecewise linear embedding $\tilde{\varphi}^{\prime}: F \rightarrow W$ such that for each $i, \tilde{\varphi}^{\prime}\left(F_{i}\right)$ has just one locally knotted point (cf. R. H. Fox and J. W. Milnor [3]). So, assume that for our imbedding $\tilde{\varphi}, \tilde{\varphi}\left(F_{i}\right)$ has always just one locally knotted point with knot type denoted by $k_{i}$.

THEOREM 2.2. Let $\delta_{1}, \ldots, \delta_{s}$ be 0 or 1 such that $\delta_{1}+\cdots+\delta_{s} \geqslant 1$. If there exists a piecewise linear imbedding $\tilde{\varphi}: F \rightarrow W$ inducing the isomorphism $\varphi_{q}$ for all $q \geqslant 0$, then $n^{\gamma}(\partial W)=2 g_{1} \delta_{1}+\cdots+2 g_{s} \delta_{s}, \quad \beta_{1}^{\gamma}(\partial W)=s-1+2 g_{1}\left(1-\delta_{1}\right)$ $+\cdots+2 g_{s}\left(1-\delta_{s}\right)$ and the induced nonsingular form $\left(\langle,\rangle^{\wedge}, t\right): \hat{T}^{1}(\partial \tilde{W}) \times$ $\hat{T}^{1}(\partial \tilde{W}) \rightarrow Q$ is cobordant to the orthogonal sum $\left(\langle,\rangle_{k_{1}} \delta_{1}, t\right) \perp \cdots \perp\left(\langle,\rangle_{k_{s}} \delta_{s}, t\right)$ and in particular $\sigma_{\omega}^{\gamma}(\partial W)=\sigma_{\omega}\left(k_{1}\right) \delta_{1}+\cdots+\sigma_{\omega}\left(k_{s}\right) \delta_{s}$ at all $\omega \in[-1,1]$ and $\sigma^{\gamma}(\partial W)=\sigma\left(k_{1}\right) \delta_{1}+\cdots+\sigma\left(k_{s}\right) \delta_{s}$, and further

$$
A_{1}^{\gamma}(t) \doteq(t-1)^{2 g_{1} \delta_{1}+\cdots+2 g_{s} \delta_{s}} \Delta_{k_{1}}\left(t^{\delta_{1}}\right) \ldots \Delta_{k_{s}}\left(t^{\delta_{s}}\right)
$$

(up to $f(t) f\left(t^{-1}\right)$ with $f(t) \in Z\langle t\rangle$ and $|f(1)|=1$ ) for all indivisible elements $\gamma \in$ $H^{1}(\partial W ; Z)$ sending $a_{1}, \ldots, a_{s}$ to $t^{\delta_{1}}, \ldots, t^{\delta_{s}}$ and independent of the subgroup $\left(1-\delta_{1}\right) G_{1}+\cdots+\left(1-\delta_{s}\right) G_{s}$.

COROllaRy 2.3. The cobordism class of the quadratic form $\left(\langle,\rangle_{k_{i}}, t\right)$ of the knot $k_{i}$ representing the locally knotted point of $\tilde{\varphi}\left(F_{i}\right)$ and its invariants: the knot signature $\sigma\left(k_{i}\right)$ and the local knot signature $\sigma_{\omega}\left(k_{i}\right)$ at all $\omega \in[-1,1]$ and the knot polynomial $\Delta_{k_{i}}(t)$ (up to $f(t) f\left(t^{-1}\right)$ with $f(t) \in Z\langle t\rangle$ and $|f(1)|=1$ ) do not depend on a particular choice of piecewise linear imbeddings inducing the isomorphism $\varphi_{q}$ for all $q>0$.

This corollary easily follows from Theorem 2.2 by taking $\delta_{1}+\cdots+\delta_{s}=1$.

2.4. Proof of Theorem 2.2. Let $N$ be a disk sum in Int $W$ of mutually disjoint regular neighborhoods $N_{i}$ of $\tilde{\varphi}\left(F_{i}\right)$ in Int $W . N_{i}$ is obtained from $F_{i}^{0} \times D^{2}$ and a 4-cell $D^{4}$ by pasting $\left(\partial F_{i}^{0}\right) \times D^{2}$ to a knotted solid torus $T_{i}$ in $\partial D^{4}$ with knot type $k_{i}$, where $F_{i}^{0}$ is a surface obtained from $\tilde{\varphi}\left(F_{i}\right)$ by removing an open 2 -cell. Since the self-intersection number of $\tilde{\varphi}\left(F_{i}\right)$ in $W$ is 0 , the framing of the knotted solid torus $T_{i}$ is the null-homologous framing. Let $a_{i}^{0}=\left[p_{i} \times \partial D\right] \in H_{1}\left(\partial N_{i} ; Z\right), i=1,2, \ldots, s$, 
where $p_{i} \in F_{i}^{0}$ and let $p_{i} \times \partial D$ have the orientation so that the linking number with $\tilde{\varphi}\left(F_{i}\right)$ in $W$ is +1 . Let $W^{\prime}=W-$ Int $N$. Clearly, $\left(W^{\prime} ; \partial W, \partial N\right)$ is a homology cobordism. Using this cobordism, the element $a_{i} \in H_{1}(\partial W ; Z)$ corresponds to the element $a_{i}^{0} \in H_{1}\left(\partial N_{i} ; Z\right)$. Without loss of generality, we can assume that $\delta_{1}$ $=\cdots=\delta_{r}=1$ and $\delta_{r+1}=\cdots=\delta_{s}=0$. Then $\gamma^{0} \in H^{1}(\partial N ; Z)$ corresponding to $\gamma$ sends $a_{1}^{0}, \ldots, a_{r}^{0}$ to $t$ and $a_{r+1}^{0}, \ldots, a_{s}^{0}$ to 1 and is independent of the subgroup $G_{r+1}^{0}+\cdots+G_{s}^{0}$, where $G_{i}^{0}$ is the image of the canonical monomorphism $i^{*}: H^{1}(N ; Z) \rightarrow H^{1}(\partial N ; Z)$ of the direct summand $H^{1}\left(N_{i} ; Z\right)$ of

$$
H^{1}(N ; Z)=H^{1}\left(N_{1} ; Z\right) \oplus \cdots \oplus H^{1}\left(N_{s} ; Z\right) \text {. }
$$

It is easily shown that $\gamma^{\prime} \in H^{1}(\partial N ; Z)$ is independent of $G_{r+1}^{0}+\cdots+G_{s}^{0}$ and $\gamma^{\prime}\left(a_{r+1}^{0}\right)=\cdots=\gamma^{\prime}\left(a_{s}^{0}\right)=1$ if and only if $\gamma^{\prime} \mid H_{1}\left(\partial N_{r+1} ; Z\right) \oplus \cdots \oplus$ $H_{1}\left(\partial N_{s} ; Z\right)=1$. [Note that $\gamma^{\prime}\left(a_{r+1}^{0}\right)=\cdots=\gamma^{\prime}\left(a_{s}^{0}\right)=1$ implies $\gamma^{\prime} \mid H_{1}\left(\partial N_{r+1} ; Z\right)$ $\oplus \cdots \oplus H_{1}\left(\partial N_{s}: Z\right) \in G_{r+1}^{0}+\cdots+G_{s}^{0}$.] Hence by direct computations, ${ }^{5}$

$$
T_{1}(\widetilde{\partial N})_{\gamma^{0}} \approx \oplus[Q\langle t\rangle /(t-1)]^{2 g_{1}+\cdots+2 g_{r}} \oplus T_{1}\left(\tilde{E}\left(k_{1}\right)\right) \oplus \cdots \oplus T_{1}\left(\tilde{E}\left(k_{r}\right)\right)
$$

and $\beta_{1}^{\gamma^{0}}(\partial N)=s-1+2 g_{r+1}+\cdots+2 g_{s}$. Further, the induced nonsingular form $\left(\langle,\rangle^{\wedge}, t\right): \hat{T}^{1}(\widetilde{\partial N}) \times \hat{T}^{1}(\widetilde{\partial N}) \rightarrow Q$ is equal to the orthogonal sum $\left(\langle,\rangle_{k_{1}}, t\right) \perp \cdots \perp\left(\langle,\rangle_{k_{r}}, t\right)$ (cf. A. Kawauchi [8, p. 578]). The desired results now follow from Corollary 1.11. This completes the proof.

EXAMPle 2.5. Consider the Matsumoto's 4-manifold $W_{1}$ (cf. Y. Matsumoto [15]) constructed as follows: Let $S^{1} \times D^{2}$ be a standard solid torus in $S^{3}$. Take the imbedding $h: S^{1} \rightarrow S^{1} \times D^{2}$ illustrated in Figure 1. Extend $h$ to a framed imbedding $\bar{h}: S^{1} \times D^{2} \rightarrow \operatorname{Int} S^{1} \times D^{2}$ so that the framing is trivial in $S^{3}$ via inclusion $S^{1} \times D^{2} \subset S^{3}$. Let $W_{1}$ be the mapping torus of $\bar{h}: W_{1}=S^{1} \times D^{2} \times[0,1] / \bar{h}$. For $g \geqslant 2$, further remove from $W_{1}$ a 4-cell $D^{4}$ which is a regular neighborhood of $p \times D^{2} \times(1 / 2), p \in S^{1}$, in $W_{1}$ meeting the boundary $\partial W_{1}$ regularly and then replace $D^{4}$ by $F_{g-1}^{0} \times D^{2}$ so that $\left(\partial F_{g-1}^{0}\right) \times D^{2}$ is identified with the unknotted solid torus $\left(W_{1}-\right.$ Int $\left.D^{4}\right) \cap D^{4}$ in $\partial D^{4}$ with trivial framing, where $F_{g-1}^{0}$ is an

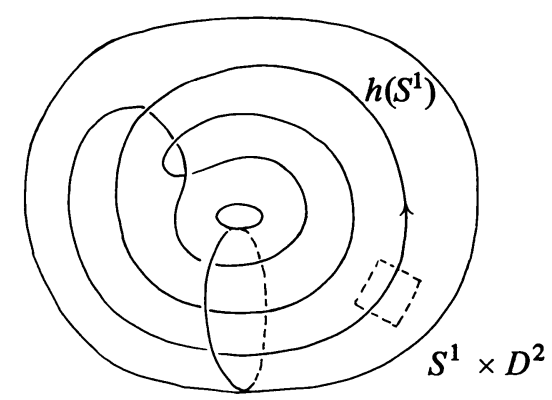

FIGURE 1

${ }^{5}$ Note the fact that

$$
H_{1}\left(\widetilde{F^{0} \times S^{1}} ; Z\right) \approx \oplus[Z\langle t\rangle /(t-1)]^{28}
$$

for all indivisible elements $\gamma \in H^{1}\left(F^{0} \times S^{1} ; Z\right)$ sending $\left[p \times S^{1}\right] \in H_{1}\left(F^{0} \times S^{1} ; Z\right)$ to $t$, where $F^{0}$ is an oriented surface of genus $g$ with an open 2-cell removed. 
oriented surface of genus $g-1$ with an open 2-cell removed. Clearly the resulting 4-manifold $W_{g}(g \geqslant 1)$ is orientable and (simple) homotopy equivalent to a surface of genus $g$. We show that there exists no piecewise linear imbedding from $F_{g}$ to $W_{g}$ inducing a homology isomorphism.

Proof. From construction the self-intersection number of a generator of $H_{2}\left(W_{g} ; Z\right)$ is 0 . So, the sequence

$$
0 \rightarrow H_{2}\left(W_{g}, \partial W_{g} ; Z\right) \stackrel{\partial}{\rightarrow} H_{1}\left(\partial W_{g} ; Z\right) \stackrel{i_{*}}{\rightarrow} H_{1}\left(W_{g} ; Z\right) \rightarrow 0
$$

is exact. Choose a basis $a, x_{1}, y_{1}, \ldots, x_{g}, y_{g}$ of $H_{1}\left(\partial W_{g} ; Z\right)$ so that $a$ is the image of a generator of $H_{2}\left(W_{g}, \partial W_{g} ; Z\right)$ by the boundary operator $\partial$ and $x_{1}$ is the homology class represented by a longitude curve in $S^{1} \times D^{2}$ which is null-homologous in $S^{3}$ - Int $S^{1} \times D^{2}$ and $y_{1}$ is a generator resulting from the mapping torus of $\bar{h}$ and the other generators $x_{2}, y_{2}, \ldots, x_{g}$ and $y_{g}$ are obtained from a homology basis for $F_{g-1}^{0} \times q, q \in \partial D^{2}$. Suppose there exists a piecewise linear imbedding $\tilde{\varphi}: F \rightarrow W$ inducing a homology isomorphism. We consider two kinds of elements $\gamma^{\prime}$ and $\gamma^{\prime \prime}$ in $H^{1}\left(\partial W_{g} ; Z\right)$ such that $\gamma^{\prime}$ sends $a, x_{1}, y_{1}$ to $t$ and $x_{2}, y_{2}, \ldots, x_{g}, y_{g}$ to 1 , and $\gamma^{\prime \prime}$ sends $a$ to $t$ and $x_{1}, y_{1}, x_{2}, y_{2}, \ldots, x_{g}, y_{g}$ to 1 . By direct calculations, ${ }^{6}$

$$
H_{1}\left(\widetilde{\partial W_{g}} ; Q\right)_{\gamma^{\prime}} \approx \oplus[Q\langle t\rangle /(t-1)]^{2 g} \oplus Q\langle t\rangle /\left(2 t^{2}-3 t+2\right)
$$

and

$$
H_{1}\left(\widetilde{\partial W_{g}} ; Q\right)_{\gamma^{\prime \prime}} \approx \bigoplus[Q\langle t\rangle /(t-1)]^{2 g} \text {. }
$$

Hence $A_{1}^{\gamma^{\prime}}(t) \doteq(t-1)^{2 g}\left(2 t^{2}-3 t+2\right)$ and $A_{1}^{\gamma^{\prime \prime}}(t) \doteq(t-1)^{2 g}$. Then by Theorem 2.2, $2 t^{2}-3 t+2$ must be of type $f(t) f\left(t^{-1}\right)(f(t) \in Z\langle t\rangle,|f(1)|=1)$, which is obviously impossible, since $2 t^{2}-3 t+2$ is irreducible. This completes the proof. ${ }^{7}$

Here is another proof. By Lemma 1.4 we have $\sigma^{\gamma^{\prime}}\left(\partial W_{g}\right)=\sigma_{3 / 4}^{\gamma^{\prime}}\left(\partial W_{g}\right)= \pm 2$ (since $\left.2 t^{2}-3 t+2 \doteq t^{2}-2(3 / 4) t+1\right)$ and $\sigma^{\prime \prime}\left(\partial W_{g}\right)=0$. This contradicts Theorem 2.2.

Using various imbeddings $S^{1} \rightarrow$ Int $S^{1} \times D^{2}$ obtained from the imbedding $h$ by tying various knots in the dotted square in Figure 1, one can obtain infinitely many 4-manifolds with similar properties.

EXAMPLE 2.6. Let $F_{g}^{0}$ be a surface obtained from a closed connected oriented surface $F_{g}$ of genus $g \geqslant 0$ by removing an open 2-cell. Taking for each positive integer $m$ a knot $k_{m}$ with Alexander polynomial $\Delta^{(m)}(t)=m t^{2}-(2 m-1) t+m$ in the boundary $\partial D^{4}$ of a 4-cell $D^{4}$, we construct a 4-manifold $W_{g}^{(m)}$ from $F_{g}^{0} \times D^{2}$ and $D^{4}$ by attaching $\left(\partial F_{g}^{9}\right) \times D^{2}$ to $D^{4}$ along a knotted solid torus in $\partial D^{4}$ with knot type $k_{m}$ and with null-homologous framing. Clearly, there is a homotopy equivalent piecewise linear imedding $\tilde{\varphi}: F_{g} \rightarrow W_{g}^{(m)}$ whose locally knotted point has

${ }^{6}$ For example, find a finite presentation of $\pi_{1}\left(\partial W_{1}\right)$ by using the van Kampen theorem and then apply [9, Corollary 2.5] for this group. For $g>1$ from construction we have

$$
H_{1}\left(\partial \tilde{W}_{g} ; Q\right)=\oplus[Q\langle t\rangle /(t-1)]^{2 g-2} \oplus H_{1}\left(\partial \tilde{W}_{1} ; Q\right)
$$

for all $\gamma \in H^{1}\left(\partial W_{g} ; Z\right)$ sending $a$ to $t$.

${ }^{7}$ The proof of the case that $n=1$ and $g=1$ gives an elementary proof of Y. Matsumoto's result [15]. 
the type $k_{m}$. However, there exists no locally flat, piecewise linear imbedding from $F_{g}$ to $W_{g}^{(m)}$ inducing a homology isomorphism.

Proof. Note that the self-intersection number of a generator of $H_{2}\left(W_{g}^{(m)} ; Z\right)$ is 0 . Let $a=\left[p \times \partial D^{2}\right] \in H_{1}\left(\partial W_{g}^{(m)} ; Z\right), p \in F_{g}^{0}$. By Theorem 2.2 we have $\sigma_{\omega}^{\gamma}\left(\partial W_{g}^{(m)}\right)=\sigma_{\omega}\left(k_{m}\right)$ at all $\omega \in[-1,1]$ and for all $\gamma \in H^{1}\left(\partial W_{g}^{(m)} ; Z\right)$ sending $a$ to $t$. Hence, by Lemma 1.4, $\sigma_{\omega}^{\gamma}\left(\partial W_{g}^{(m)}\right)= \pm 2$ if $\omega=(2 m-1) / 2 m$ and $\sigma_{\omega}^{\gamma}\left(\partial W_{g}^{(m)}\right)=0$ if $\omega \neq(2 m-1) / 2 m$ (cf. J. W. Milnor [19]). By Theorem 2.2 there exists no locally flat piecewise linear imbedding from $F_{g}$ to $W_{g}^{(m)}$ inducing a homology isomorphism. This completes the proof.

Further, we obtain that no two manifolds in the 4-manifolds $W_{g}^{(m)}, m=$ $1,2,3, \ldots$ are relatively homology cobordant. ${ }^{8}$ To see this, note that by Theorem $2.2 \sigma_{\omega}^{\gamma}\left(\partial W_{g}^{(m)}\right)$ at each $\omega \in[-1,1]$ is invariant under all $\gamma \in H^{1}\left(\partial W_{g}^{(m)} ; Z\right)$ sending $a$ to $t^{ \pm 1}$ and hence that $\sigma_{\omega}^{\gamma}\left(\partial W_{g}^{(m)}\right)$ at each $\omega \in[-1,1]$ is invariant under relative homology cobordisms. It follows from the above calculation of the local signature that no two manifolds are relatively homology cobordant.

Of course, any $W_{g}^{(m)}$ is not relatively homology cobordant to $F_{g} \times D^{2}$.

EXAMPLE 2.7. Consider a link $L_{0}$ of 2-components illustrated in Figure 2 in a 3-cell $D^{3} \subset S^{3}$. For each $s \geqslant 2$, let $L$ be a link of $s$ components in $S^{3}$ obtained from $L_{0}$ by adding a trivial (i.e. unknotted and unlinked) link of $(s-2)$ components in $S^{3}-D^{3}$. (If $s=2$, then $L=L_{0}$.) Clearly, this link $L$ is a boundary link (that is, the components of $L$ bound mutually disjoint orientable surfaces in $S^{3}$ ). Let $e_{1}, e_{2}, \ldots, e_{s}$ be a basis of $H_{2}\left(W_{L} ; Z\right)$ coming from the components of $L$, where $e_{1}, e_{2}$ come from $L_{0}$. We show that $e_{1}, e_{2}, \ldots, e_{s}$ are not represented by mutually disjoint piecewise linearly imbedded 2-spheres.

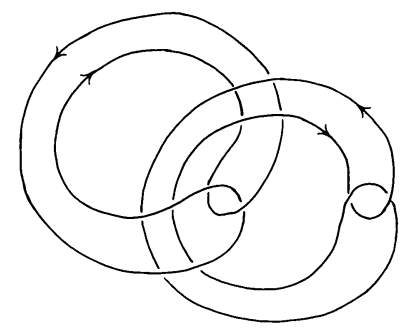

FIGURE 2

Proof. Let $a_{1}, \ldots, a_{s}$ be a basis of $H_{1}\left(\partial W_{L} ; Z\right)$ dually related to the basis $e_{1}, \ldots, e_{s}$ of $H_{2}\left(W_{L} ; Z\right)$. Let $\gamma, \gamma_{1}$ and $\gamma_{2}$ be the indivisible elements of $H^{1}\left(\partial W_{L} ; Z\right)$ such that $\gamma=\gamma_{1}+\gamma_{2}$ and $\gamma_{i}\left(a_{i}\right)=t$ and $\gamma_{i}\left(a_{j}\right)=1, i \neq j, i=1,2$. Suppose the basis $e_{1}, \ldots, e_{s}$ are represented by $s$ mutually disjoint piecewise linearly imbedded 2-spheres in $W_{L}$ with locally knotted points of types $k_{1}, \ldots, k_{s}$. By direct computations, ${ }^{9} A_{1}^{\gamma}(t) \doteq\left(t^{2}-t+1\right)\left(t^{2}-3 t+1\right), A_{1}^{\gamma_{i}}(t) \doteq 1, i=1,2$.

\footnotetext{
${ }^{8}$ Two bounded oriented manifolds $W_{1}$ and $W_{2}$ are relatively homology cobordant, if there is a relative oriented cobordism $\bar{W}$ between $W_{1}$ and $W_{2}$ such that $H_{*}\left(\bar{W}, W_{i} ; Z\right)=H_{*}\left(\partial \bar{W}, \partial W_{i} ; Z\right)=0, i=1,2$.

${ }^{9}$ Compute a finite presentation of $\pi_{1}\left(\partial W_{L}\right)$ by using the van Kampen theorem and then apply [9, Corollary 2.5].
} 
By Lemma 1.4 and Theorem 2.2, $\sigma_{1 / 2}\left(k_{1}\right)+\sigma_{1 / 2}\left(k_{2}\right)=\sigma_{1 / 2}^{\gamma}\left(\partial W_{L}\right)= \pm 2$ and $\sigma_{1 / 2}\left(k_{i}\right)=\sigma_{1 / 2}^{\gamma_{i}}\left(\partial W_{L}\right)=0, i=1,2$, a contradiction.

In particular, this boundary link $L$ is not cobordant to a completely splittable link (i.e. a link whose components $C_{1}, \ldots, C_{s}$ are contained in mutually disjoint 3-cells $B_{1}, B_{2}, \ldots, B_{s}$ in $S^{3}$ so that $\left.C_{i} \subset B_{i}, i=1, \ldots, s\right)$.

To derive a further property of a piecewise linear imbedding of 2 -spheres into a 4-manifold, let $F$ be a surface consisting of $s$ 2-spheres $S_{1}, \ldots, S_{s}$ and let $W$ be a compact connected oriented 4-manifold with $H_{q}(W ; Q) \approx H_{q}(F ; Q)$ for all $q>0$ and such that any two elements of $H_{2}(W ; Z) /($ torsions) have the intersection number 0 . Then $\partial W$ is connected and we have an isomorphism

$$
\partial: H_{2}(W, \partial W ; Q) \approx H_{1}(\partial W ; Q),
$$

so $H_{1}(\partial W ; Z)$ has the rank $s$.

THEOREM 2.8. If there exists a piecewise linear imbedding $\tilde{\varphi}: F \rightarrow W$ inducing $a$ monomorphism $\tilde{\varphi}_{*}: H_{q}(F ; Z) \rightarrow H_{q}(W ; Z)$ for all $q>0$, then we have $\beta_{1}^{\gamma}(\partial W)=s$ -1 and $A_{1}^{\gamma}(1) \neq 0$ for all nonzero elements $\gamma \in H^{1}(\partial W ; Z)$ (cf. [9, Theorem C]).

Proof. By Lemma 1.1 (the reduction formula), it suffices to prove this theorem for all indivisible elements $\gamma \in H^{1}(\partial W ; Z)$. Let $\gamma \in H^{1}(\partial W ; Z)$ be an indivisible element. We can find a basis $b_{1}, \ldots, b_{s}$ of $H_{1}(\partial W ; Z) /$ (torsions) such that $\gamma\left(b_{i}\right)=t, i=1, \ldots, s$. Let $N$ be a disk sum in Int $W$ of mutually disjoint regular neighborhoods $N_{i}$ of $S_{i}$ in Int $W$. Since the self-intersection number of $S_{i}$ in $W$ is 0 , $H_{1}\left(\partial N_{i} ; Z\right)=Z$, that is, $\partial N_{i}$ is a homology orientable handle (cf. [11]). Note that $\partial N$ is homeomorphic to the connected sum $\left(\partial N_{1}\right) \#\left(\partial N_{2}\right) \# \cdots \#\left(\partial N_{s}\right)$. Let $W^{\prime}=$ $W$ - Int $N$. Clearly, $\left(W^{\prime} ; \partial W, \partial N\right)$ is a rational homology cobordism. Note that $I$ : $H_{1}(\partial W ; Z) /($ torsions $) \rightarrow H_{1}\left(W^{\prime} ; Z\right) /$ (torsions) induced by inclusion is a monomorphism of a finite index. There are bases $\left\{x_{1}, \ldots, x_{s}\right\},\left\{y_{1}, \ldots, y_{s}\right\}$ for $H_{1}(\partial W ; Z) /\left(\right.$ torsions), $H_{1}\left(W^{\prime} ; Z\right) /($ torsions), respectively, and nonzero integers $n_{1}, \ldots, n_{s}$ such that $I\left(x_{i}\right)=n_{i} y_{i}, i=1,2, \ldots, s$. Let $n$ be the least common multiple of $n_{1}, \ldots, n_{s}$ and let $n=n_{i} \tilde{n}_{i}$. Further let $\left(m_{i j}\right)$ be an invertible matrix with $\left(b_{1}, \ldots, b_{s}\right)=\left(x_{1}, \ldots, x_{s}\right)\left(m_{i j}\right)$. Let $d_{1}, \ldots, d_{s}$ be integers such that $(1, \ldots, 1)=\left(d_{1}, \ldots, d_{s}\right)\left(m_{i j}\right)$. Define a map $\bar{\gamma}: H_{1}\left(W^{\prime} ; Z\right) /($ torsions $) \rightarrow\langle t\rangle$ by the identities $\bar{\gamma}\left(y_{i}\right)=t^{\tilde{n}_{i} d_{i}}, i=1, \ldots, s$. Let $\gamma_{n}=\bar{\gamma} I: H_{1}(\partial W ; Z) /($ torsions $) \rightarrow\langle t\rangle$. We have

$$
\gamma_{n}\left(b_{j}\right)=\gamma_{n}\left(\sum_{i} m_{i j} x_{i}\right)=\bar{\gamma}\left(\sum_{i} m_{i j} n_{i} y_{i}\right)=t^{\left(\Sigma_{i} m_{i j} d_{i}\right) n}=t^{n}
$$

$j=1, \ldots, s$. By Lemma 1.1 it suffices to prove the assertion for this map $\gamma_{n}$. By Corollary $1.14, \beta_{1}^{\gamma_{n}}(\partial W)=\beta_{1}^{\gamma^{\prime}}(\partial N)$, and $A_{1}^{\gamma_{n}}(1) \neq 0$ is equivalent to $A_{1}^{\gamma^{\prime}}(1) \neq 0$, where $\gamma^{\prime}$ is the restriction of $\bar{\gamma}$ to $\partial N$. Since $\partial N$ is the connected sum of the homology orientable handles $\partial N_{i}, i=1, \ldots, s$, it is not difficult to see that $\beta_{1}^{\gamma^{\prime \prime}}(\partial N)=s-1$ and $A_{1}^{\gamma^{\prime \prime}}(1) \neq 0$ for all indivisible elements $\gamma^{\prime \prime} \in H^{1}(\partial N ; Z) .{ }^{10}$ It follows from Lemma 1.1 that $\beta_{1}^{\gamma_{n}}(\partial W)=\beta_{1}^{\gamma^{\prime}}(\partial N)=s-1$ and $A_{1}^{\gamma_{n}}(1) \neq 0$. This completes the proof.

\footnotetext{
${ }^{10}$ Note that there is a homomorphism from $\pi_{1}(\partial N)$ onto the free group of rank $s$.
} 
Let $L$ be a link of $s(\geqslant 2)$ components. $\beta_{1}(L)$ is defined to be $\beta_{1}^{\gamma^{0}}(E(L))$, where $E(L)$ is a 3-manifold obtained from $S^{3}$ by removing an open tubular neighborhood of $L$ and $\gamma^{0} \in H^{1}(E(L) ; Z)$ is an indivisible element sending each oriented meridian curve of $L$ to $t$ (cf. [9]). We have that $\beta_{1}(L) \leqslant s-1$. (See [10, Corollary 2.3].) Suppose $L$ has the linking numbers 0 .

Corollary 2.9. If $\beta_{1}(L) \leqslant s-2$, then any $s$ elements of $H_{2}\left(W_{L} ; Z\right)$ forming a basis of $H_{2}\left(W_{L} ; Q\right)$ cannot be represented by mutually disjoint, piecewise linearly imbedded 2-spheres.

Proof. We may consider that $\partial W_{L}$ is obtained from $E(L)$ and $s$ solid tori $T_{1}, \ldots, T_{s}$ by attaching $\partial T_{i}$ to one component of $\partial E(L)$. From the construction of $W_{L}$ we have an isomorphism $i_{*}: H_{1}(E(L) ; Z) \approx H_{1}\left(\partial W_{L} ; Z\right)$. Hence the indivisible element $\gamma^{0} \in H^{1}(E(L) ; Z)$ canonically determines an indivisible element $\gamma \in$ $H^{1}\left(\partial W_{L} ; Z\right)$. Take an infinite cyclic cover $\left(\widetilde{W}_{L}, \tilde{E}(L)\right)$ associated with $\gamma$. Since

$$
H_{2}\left(\partial \widetilde{W}_{L}, \tilde{E}(L) ; Q\right) \rightarrow H_{1}(\tilde{E}(L) ; Q) \stackrel{i_{*}}{\rightarrow} H_{1}\left(\widetilde{\partial W}_{L} ; Q\right) \stackrel{j_{*}}{\rightarrow} H_{1}\left(\widetilde{\partial W}_{L}, \tilde{E}(L) ; Q\right)
$$

is exact and, by excision, $H_{*}\left(\partial \widetilde{W}_{L}, \tilde{E}(L) ; Q\right)=\bigoplus_{i=1}^{s} H_{*}\left(\tilde{T}_{i}, \partial \tilde{T}_{i} ; Q\right)$ is a $Q\langle t\rangle-$ torsion module, it follows that $\beta_{1}(L)=\beta_{1}^{\gamma}\left(\partial W_{L}\right)$. By Theorem 2.8 we obtain a desired result.

EXAMPLE 2.10. Consider the Whitehead link $L_{0}$ (of 2-components) (see, for example, [9, Figure 1]) and the links $Q_{0}$ and $E_{0}$ illustrated in Figure 3. By direct computations, ${ }^{11} \beta_{1}\left(L_{0}\right)=\beta_{1}\left(Q_{0}\right)=\beta_{1}\left(E_{0}\right)=0$. Let $L^{2}$ be $L_{0}, Q_{0}$ or $E_{0}$. For each $s \geqslant 3$, let $L^{s}$ be a link of $s$ components obtained from the link $L^{2}$ in a 3-cell $D^{3} \subset S^{3}$ by adding a trivial link of $(s-2)$ components in $S^{3}-D^{3}$. We obtain $\beta_{1}\left(L^{s}\right)=s-2$, since $\beta_{1}\left(L^{2}\right)=0$. By Corollary 2.9 , for each $s \geqslant 2$ any s elements of $H_{2}\left(W_{L^{s}} ; Z\right)$ forming a basis of $H_{2}\left(W_{L^{s}} ; Q\right)$ cannot be represented by mutually disjoint piecewise linearly imbedded 2-spheres. For $L^{2}=L_{0}$ we obtain a stronger assertion than a result in [9, Application 3]. For $L^{2}=Q_{0}$ or $E_{0}$ this answers enough a question of $\mathrm{H}$. Lambert [13] who asked whether either of the links $Q_{0}, E_{0}$ bounds two 2-cells mutually-disjointly and piecewise linearly imbedded in $D^{4}$. By applying, more directly, Theorem 2.8 we have that the link $L^{s}$ cannot bound $s$ 2-cells mutually-disjoint and piecewise linearly imbedded in any rational homology 4-cell.

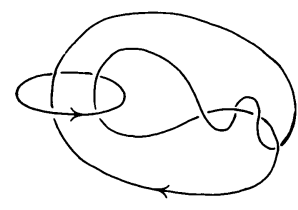

$Q_{0}$

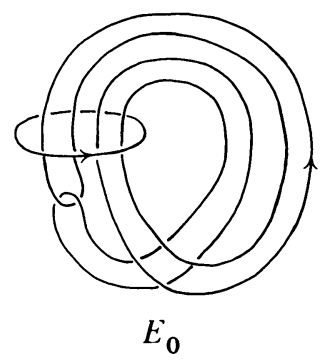

FIGURE 3

${ }^{11}$ See [9, Corollary 2.4]. 
One may note that Corollary 2.9 cannot apply for boundary links, since $\beta_{1}(L)=$ $s-1$ for all boundary links $L$ of $s$ components (cf. N. Smythe [22] and [9, Corollary 2.4]).

Now suppose $F$ and $W$ satisfy the assumptions of Theorem 2.8. For an integer $n$ and an element $\gamma \in H^{1}(\partial W ; Z)$ we let $\gamma_{n}=n \gamma \in H^{1}(\partial W ; Z)$. The proof of Theorem 2.8 combined with Corollary 1.14 also implies the following.

COROLlaRy 2.11. If there exists a locally flat, piecewise linear imbedding $\tilde{\varphi}$ : $F \rightarrow W$ inducing a monomorphism $\tilde{\varphi}_{*}: H_{q}(F ; Z) \rightarrow H_{q}(W ; Z)$ for all $q>0$, then for each indivisible element $\gamma \in H^{1}(\partial W ; Z)$ there exists a positive integer $n$ such that $\sigma_{\omega}^{\gamma_{n}}(\partial W)=0$ at all $\omega \in(-1,1]$ and $A_{1}^{\gamma_{n}}(t) \doteq f(t) f\left(t^{-1}\right)$ for some $f(t) \in Q\langle t\rangle$ with $f(1) \neq 0$.

EXAMPLE 2.12. Let $W_{0}^{(m)}$ be a 4-manifold in Example 2.6 with $g=0 . W_{0}^{(m)}$ is homotopy equivalent to $S^{2}$ and any element of $H_{2}\left(W_{0}^{(m)} ; Z\right)$ can be represented by a piecewise linearly imbedded 2-sphere. However, any nonzero element of $H_{2}\left(W_{0}^{(m)} ; Z\right)$ cannot be represented by a locally flat 2-sphere.

Proof. $H_{1}\left(\widetilde{\partial W}_{0}^{(m)} ; Z\right)_{\gamma_{n}} \approx Q\langle t\rangle /\left(\Delta^{(m)}\left(t^{n}\right)\right), \Delta^{(m)}\left(t^{n}\right)=m t^{2 n}-(2 m-1) t^{n}+m$ by Lemma 1.1. $\Delta^{(m)}\left(t^{n}\right)$ has $2 n$ distinct roots of complex numbers of norm 1. [Take the derivative $d \Delta^{(m)}\left(t^{n}\right) / d t$.] Let $t^{2}-2 \omega t+1,-1<\omega<1$, be any real irreducible factor of $\Delta^{(m)}\left(t^{n}\right)$. We have $\sigma_{\omega}^{\gamma_{n}}\left(\partial W_{0}^{(m)}\right)= \pm 2$. Therefore by Corollary 2.11 , we obtain a desired result.

This fact was also obtained by M. Kato [5, Theorem D]. ${ }^{12}$

Let ${ }_{s} W^{(m)}$ be a disk sum of $W_{0}^{(m)}$ and $(s-1)$ copies of $S^{2} \times D^{2}$. By Corollary 2.11 the above proof also implies that any $s$ elements of $H_{2}\left({ }_{s} W^{(m)} ; Z\right)$ forming $a$ basis for $H_{2}\left({ }_{s} W^{(m)} ; Q\right)$ cannot be represented by mutually disjoint locally flat 2-spheres.

\section{REFERENCES}

1. S. E. Cappell and J. L. Shaneson, Totally spineless manifolds, Illinois J. Math. 21 (1977), 231-239.

2. W. T. Eaton, C. P. Pixley and G. A. Venema, A topological embedding which cannot be approximated by a piecewise linear embedding, Notices Amer. Math. Soc. 24 (1977), A-302.

3. R. H. Fox and J. W. Milnor, Singularities of 2-spheres in 4-space and cobordism of knots, Osaka J. Math. 3 (1966), 257-267.

4. C. H. Giffen, Disciplining dunce hats in 4-manifolds (preprint).

5. M. Kato, Embeddings of spheres and balls in codimension < 2, Invent. Math. 10 (1970), 89-107.

6. A. Kawauchi, On links not cobordant to split links (preprint).

7. A partial Poincare duality theorem for infinite cyclic coverings, Quart. J. Math. Oxford Ser. 26 (1975), 437-458.

8.,$\tilde{H}$-cobordism. I: The group among three dimensional homology handles, Osaka J. Math. 13 (1976), 567-590.

9. , On quadratic forms of 3-manifolds, Invent. Math. 43 (1977), 177-198.

10. __ On the Alexander polynomials of cobordant links, Osaka J. Math. 15 (1978), 151-159.

\footnotetext{
${ }^{12}$ We can also obtain a result of Theorem $D$ in the higher even dimensional case as an analogy of the above proof by using Corollary 1.14 and considering a $(2 r-1)$-knot with the $r$ th Alexander polynomial $m t^{2}-(2 m-1) t+m$ or $m t^{4}-(2 m-1) t^{2}+m$ according as $r$ is odd or even. Note that Kato's proof contains some erroneous point. [Let $W$ be a compact connected orientable $(2 r+2)$-manifold with $H_{*}(W ; Q) \approx H_{*}\left(S^{1} ; Q\right)$ and such that $H_{*}(\partial W ; Z) \approx H_{*}\left(S^{1} \times S^{2 r} ; Z\right)$. The $r$ th Alexander polynomial of $\partial W$ with a generator $\gamma \in H^{1}(\partial W ; Z)$ is not necessarily of a slice type.]
} 
11. __ Three dimensional homology handles and circles, Osaka J. Math. 12 (1975), 565-581.

12. ___ Imbedding problem of 3-manifolds into 4-manifolds (in preparation).

13. H. Lambert, $A$ class of non-splittable links, Michigan Math. J. 24 (1977), 45-50.

14. J. Levine, Invariants of knot cobordism, Invent. Math. 8 (1969), 98-110.

15. Y. Matsumoto, A 4-manifold which admits no spine, Bull. Amer. Math. Soc. 81 (1975), 467-470.

16. Some counterexamples in the theory of embedding manifolds in codimension two, Sci. Papers

College Gen. Ed. Univ. Tokyo 25 (1975), 49-57.

17. Secondary intersectional properties of 4-manifolds and Whitney's trick, Proc. Sympos. Pure Math., vol. 32, Amer. Math. Soc., Providence, R. I., 1978, pp. 99-107.

18. Wild embeddings of piecewise linear manifolds in codimension two (preprint).

19. J. W. Milnor, Infinite cyclic coverings, Conference on the Topology of Manifolds, Prindle, Weber \& Schmidt, Boston, Mass., 1968.

20. On isometries of inner product spaces, Invent. Math. 8 (1969), 83-97.

21. K. Murasugi, On a certain numerical invariant of link types, Trans. Amer. Math. Soc. 117 (1967), $387-422$.

22. N. Smythe, Boundary links, Topology Seminar, Wisconsin, 1965, Ann. Math. Studies, no. 60, Princeton Univ. Press, Princeton, N. J., 1966, pp. 69-72.

School of Mathematics, The Institute for Advanced Study, Princeton, New Jersey 08540

Department of Mathematics, Osaka City University, Osaka, Japan 